\title{
An Image Steganography-based Novel Approach to develop 8-Share Integrated Security Toolkit (ISTI-8)
}

\author{
Sabyasachi Samanta \\ Haldia Institute of Technology, Haldia, WB, INDIA \\ E-mail id: sabyasachi.smnt@gmail.com \\ Saurabh Dutta \\ Dr. B. C. Roy Engineering College, Durgapur, WB, INDIA \\ E-mail id: saurabh.dutta@bcrec.org \\ Gautam Sanyal \\ National Institute of Technology, Durgapur, WB, INDIA \\ E-mail id: nitgsanyal@gmail.com
}

\begin{abstract}
Encryption is a process or algorithm to make information hidden or secret and considered as a subset of cryptography. Using encryption data are being transformed into some another form that appears to be meaningless and incomprehensible. Here we have embedded encrypted data bits about the entire image to some suitable nonlinear pixel positions using key. After that we have formed several shares of image and key using $\mathrm{R}, \mathrm{G}$ and $\mathrm{B}$ components and character or digits respectively. At the decryption end through appropriate arrangement of shares of image and key, make possible to retrieve hidden data bits from stego-image and reform into its original content.
\end{abstract}

Index Terms - Steganography, Nonlinear Pixel Position (NPP), Modulus Division based Encryption (MDE), Visual Cryptography, Component based Visual Cryptography for Image (CVCI), Integrated Security Toolkit for Image (ISTI).

\section{INTRODUCTION}

Encryption is the process for transforming plaintext into the cipher text. Where plaintext is the input and cipher text is the output of the encryption process. Decryption is the process of transforming cipher text into the plaintext. Where cipher text is the input and plaintext is the output of the decryption process. Visual cryptography is an emerging cryptography technology, uses the characteristics of human vision to decrypt encrypted images. It needs neither cryptography knowledge nor the complex computation [1][14]. In encryption procedure, it uses the technique for hiding a two-tone secret image into a set of binary transparencies which seem like random noise. The visual cryptography uses secret sharing scheme based on a $\{k, n\}$ threshold framework, where $n$ means a secret image will be hidden in $n$ transparencies, and $\mathrm{k}$ is that we can stack $k$ or more than $k$ transparencies to reconstruct the secret image in visual[1] [14].

In this paper, we have proposed a technique to embed the encrypted (using MDE method) message about the entire image at arbitrary pixel positions using NPP-2 bit method [3]. The text taken from the keyboard or special characters encoded into its ASCII-8 (American Standard Code for Information Interchange) binary equivalent. Here we also have taken a key $(\mathrm{K})$ with 6-digit. Then the corresponding ASCII-8 value is taken for each character or digit and added each of it. The total key value is divided by eight and we get reminder. Depending on the reminder the encryption is being processed (Figure 2). At the time of encryption three bits are taken from the data array and replaced as per encryption wheel. Each three bit block is replaced by encryption circle. The encrypted data bits again divided into 8-bit blocks and $\mathrm{n}^{\text {th }}$ bit left or right shifted. If the reminder be odd then the left shift is performed. Otherwise the right shift is performed. If reminder is zero, then no shifting is being performed. At the time of embedding a pair off encrypted data bits are taken and rooted to carrier image at nonlinear pixel position (NPP) through the key and we get stego-image cascading unique methods i.e. through Integrated Security Toolkit for Image (ISTI). Finally, we have made different image and key shares using CVCI method as in Table1. In figure 1 the flow diagram for ISTI method has been depicted. 


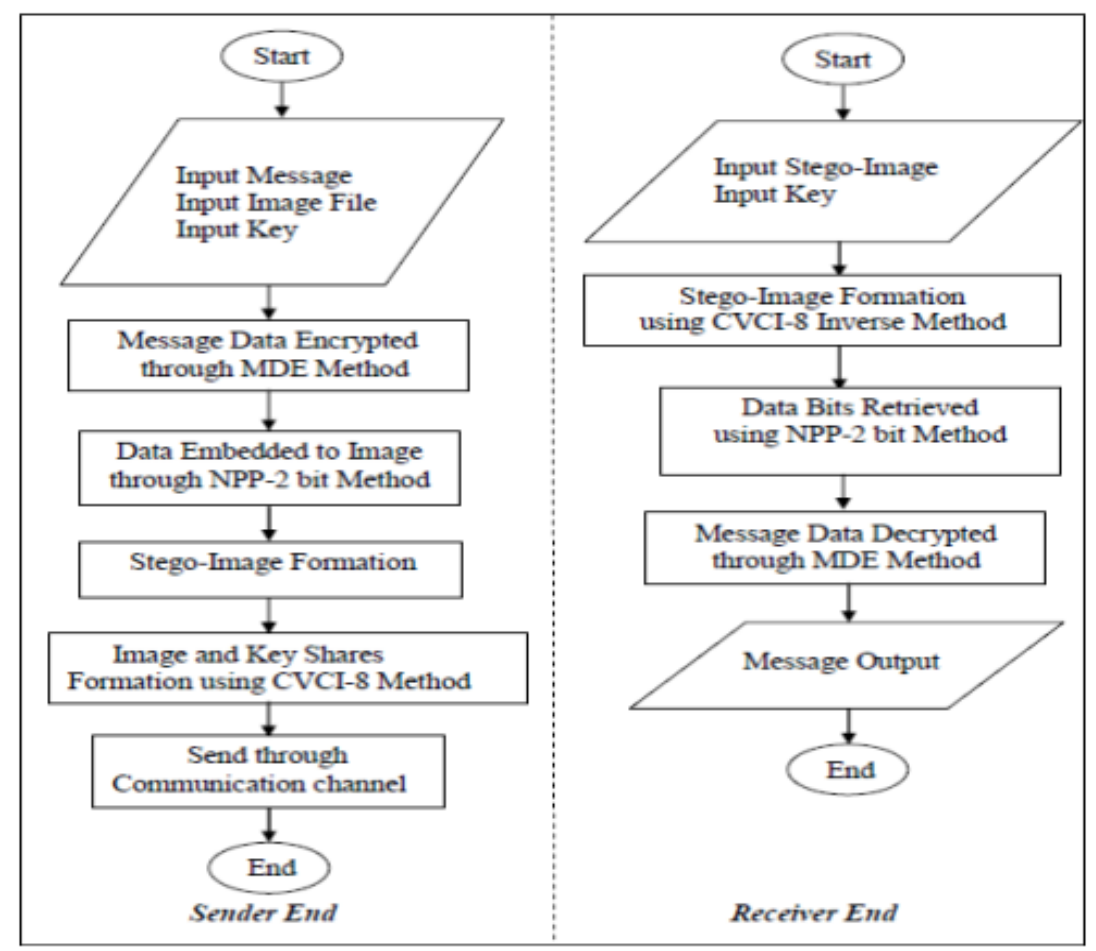

Fig.1. Flow Diagram for ISTI Method

In our work, we have targeted any two bit of last five significant bit of each R, G and B of any selected random pixel positions. The replacement of all the bits have done in nonlinear pixel and bit positions, in any one of five significant bit of $R, G$ and $B$ at selected pixels about the entire image using the private key cryptography technique taking the $\alpha$ value as 255 or as in the original image[2][3]. As, we have altered only any two bit of last five significant bits. If any bit generated from text become same to the targeted bit of image then there will be no change i.e. it will produce the same to original image [4] [5] [10].

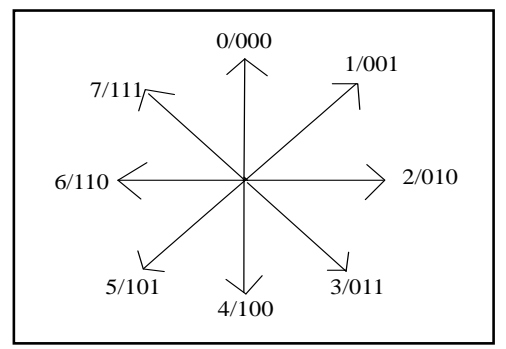

Fig.2. Encryption wheel

Here 8-image shares ( $\left.\mathrm{IS}_{0}-\mathrm{IS}_{7}\right)$ are formed using presence $(\mathrm{P})$ or absence $(\mathrm{A})$ of Red $\left(\mathrm{IM}_{\mathrm{R}}\right)$, Green $\left(\mathrm{IM}_{\mathrm{G}}\right)$ and Blue $\left(\mathrm{IM}_{\mathrm{B}}\right)$ color components respectively. Also the key shares $\left(\mathrm{KS}_{0}-\mathrm{KS}_{7}\right)$ are formed with presence $(\mathrm{P})$ or absence (A) of key shares $\left(\mathrm{K}_{1}, \mathrm{~K}_{2}\right.$ and $\left.\mathrm{K}_{3}\right)$. At the decryption end suitable combination of image and key shares make possible to reform the stego-image (SI) and key $(\mathrm{K})$. Formation of stego-image by using image shares $\left(\mathrm{IM}_{\mathrm{R}}, \mathrm{IM}_{\mathrm{G}}\right.$ and $\left.\mathrm{IM}_{\mathrm{B}}\right)$ :

By using only one share: $\mathrm{SI}_{11}=\left\{\mathrm{IS}_{7}\right\}$
By using only two shares:

$$
\begin{array}{ll}
\mathrm{SI}_{21}=\left\{\mathrm{IS}_{0}, \mathrm{IS}_{7}\right\} & \mathrm{SI}_{22}=\left\{\mathrm{IS}_{1}, \mathrm{IS}_{6}\right\} \\
\mathrm{SI}_{23}=\left\{\mathrm{IS}_{2}, \mathrm{IS}_{5}\right\} & \mathrm{SI}_{24}=\left\{\mathrm{IS}_{3}, \mathrm{IS}_{4}\right\} \\
\mathrm{SI}_{25}=\left\{\mathrm{IS}_{3}, \mathrm{IS}_{5}\right\} & \mathrm{SI}_{26}=\left\{\mathrm{IS}_{5}, \mathrm{IS}_{6}\right\} \\
\mathrm{SI}_{27}=\left\{\mathrm{IS}_{3}, \mathrm{IS}_{6}\right\} &
\end{array}
$$

Presence of shares $\mathrm{IS}_{7}$ (similar to $\mathrm{S}_{21}$ \} may produce more six combination like $\left\{\left\{\mathrm{IS}_{1}, \mathrm{IS}_{7}\right\}, \quad\left\{\mathrm{IS}_{2}, \mathrm{IS}_{7}\right\}\right.$, $\left.\left\{\mathrm{IS}_{3}, \mathrm{IS}_{7}\right\},\left\{\mathrm{IS}_{4}, \mathrm{IS}_{7}\right\},\left\{\mathrm{IS}_{5}, \mathrm{IS}_{7}\right\},\left\{\mathrm{IS}_{6}, \mathrm{IS}_{7}\right\}\right\}$. For the case of image, the shares $\mathrm{S}_{0}$ forms a $100 \%$ black image and $\mathrm{IS}_{7}$ form the stego-image. So the presence of $\mathrm{IS}_{7}$ means the cent percent presence of stego-image.

Table 1. Formation of Image and Key Subset Using Shares

\begin{tabular}{|c|c|c|c|c|c|c|}
\hline $\begin{array}{c}\text { Image(IS)/ } \\
\text { Key(KS) } \\
\text { Subset }\end{array}$ & \multicolumn{3}{|c|}{ Image Shares } & \multicolumn{3}{c|}{ Key Shares } \\
\cline { 2 - 7 } & $\mathrm{IM}_{\mathrm{R}}$ & $\mathrm{IM}_{\mathrm{G}}$ & $\mathrm{IM}_{\mathrm{B}}$ & $\mathrm{K}_{1}$ & $\mathrm{~K}_{2}$ & $\mathrm{~K}_{3}$ \\
\hline $\mathrm{S}_{0}$ & $\mathrm{~A}$ & $\mathrm{~A}$ & $\mathrm{~A}$ & $\mathrm{~A}$ & $\mathrm{~A}$ & $\mathrm{~A}$ \\
\hline $\mathrm{S}_{1}$ & $\mathrm{~A}$ & $\mathrm{~A}$ & $\mathrm{P}$ & $\mathrm{A}$ & $\mathrm{A}$ & $\mathrm{P}$ \\
\hline $\mathrm{S}_{2}$ & $\mathrm{~A}$ & $\mathrm{P}$ & $\mathrm{A}$ & $\mathrm{A}$ & $\mathrm{P}$ & $\mathrm{A}$ \\
\hline $\mathrm{S}_{3}$ & $\mathrm{~A}$ & $\mathrm{P}$ & $\mathrm{P}$ & $\mathrm{A}$ & $\mathrm{P}$ & $\mathrm{P}$ \\
\hline $\mathrm{S}_{4}$ & $\mathrm{P}$ & $\mathrm{A}$ & $\mathrm{A}$ & $\mathrm{P}$ & $\mathrm{A}$ & $\mathrm{A}$ \\
\hline $\mathrm{S}_{5}$ & $\mathrm{P}$ & $\mathrm{A}$ & $\mathrm{P}$ & $\mathrm{P}$ & $\mathrm{A}$ & $\mathrm{P}$ \\
\hline $\mathrm{S}_{6}$ & $\mathrm{P}$ & $\mathrm{P}$ & $\mathrm{A}$ & $\mathrm{P}$ & $\mathrm{P}$ & $\mathrm{A}$ \\
\hline $\mathrm{S}_{7}$ & $\mathrm{P}$ & $\mathrm{P}$ & $\mathrm{P}$ & $\mathrm{P}$ & $\mathrm{P}$ & $\mathrm{P}$ \\
\hline
\end{tabular}

For the key formation, by using (same as for stegoimage) the key shares $\left(\mathrm{KS}_{1}, \mathrm{KS}_{2}, \mathrm{KS}_{3}\right)$ we may get back the original key $(\mathrm{K})$. Also the presence of $\mathrm{KS}_{7}$ means the cent percent presence of key $(\mathrm{K})$.

Section 2 represents the scheme followed in the encryption technique. Section 3 represents an implementation of the technique. Section 4 gives you an 
idea about the experimental results. Section 5 is an analytical discussion on the technique. Section 6 draws a conclusion.

\section{RELATED WORKS}

In this section we have discussed various encryption and steganographic data hiding methods.

\section{A. Encryption methods}

1. Encryption Algorithms are divided into 2 categories as follows:-

a) Symmetric Key Encryption - In symmetric key encryption technique, single key is used in both encryption and decryption procedure. The key must be known to both the sender and receiver before encryption or decryption. So, the secret key plays important role and its strength depends on the length of key (in bits). Symmetric key encryption algorithms are- RC2, DES, 3DES, RC5, Blowfish, and AES et al [12].

b) Asymmetric Key Encryption - Asymmetric key encryption algorithm uses two types of keys, Private keys and Public Keys. Public Key is used to encrypt the original data or plaintext and generate a cipher text [13]. This cipher text is decoded by the receiver when it receives, by using its own Private Key. Private Key is also known as secret key because it is unknown to all. Or it's known only to the person, who receives it or can say authorized person. Asymmetric key encryption algorithms are RSA, Digital Signatures et al.

\section{Steganographic methods:}

\section{A. Spatial Domain Steganographic Method}

a) Data Hiding by LSB: Various techniques about data hiding have been proposed in literatures. One of the common techniques is based on manipulating the least-significant-bit (LSB)[4][8] and [9] planes by directly replacing the LSBs of the cover-image with the message bits.

b) Data Hiding by PVD: The pixel-value differencing (PVD) method proposed by $\mathrm{Wu}$ and Tsai [6] can successfully provide both high embedding capacity and outstanding imperceptibility for the stego-image. The pixelvalue differencing (PVD) method segments the cover image into non overlapping blocks containing two connecting pixels and modifies the pixel difference in each block (pair) for data embedding.

c) Data Hiding by GLM: In 2004, Potdar et al.[7] proposes GLM (Gray level modification) technique which is used to map data by modifying the gray level of the image pixels. Gray level modification Steganography is a technique to map data (not embed or hide it) by modifying the gray level values of the image pixels. GLM technique uses the concept of odd and even numbers to map data within an image.

\section{THE SCHEME}

This section represents a description of the actual scheme used during "An Image Steganography-based Novel Approach to develop 8-Share Integrated Security Toolkit (ISTI-8)" technique. Section 3.1 describes the encryption technique using five algorithms 3.1.1, 3.1.2 \& 3.1.3 while section 3.2 describes the decryption technique using algorithm 3.2.1 [3].

\subsection{Encryption of data bits about the image and formation of image shares using ISTV-8 method}

\subsubsection{Create an encrypted array using MDE method}

Step I: Take text input from keyboard or special characters and calculate the string length (chlen).

Step II: Convert the length (chlen) into its 8 bit binary equivalent. Store that data bits to earr[bit] as LSB (Least Significant Bit) to Arr[1] and MSB (Most Significant Bit) to Arr[8] respectively.

Step III: Convert each character of text into its ASCII8 binary equivalent.

Step IV: Taking the key input, the corresponding ASCII-8 value of each character or digits is added.

Step V: Depending on the reminder through the modulus division by eight each three bit of Arr[] is encrypted.

Step VI: Again depending on the reminder the data bits are left or right shifted.

Step VII: Stop.

\subsubsection{Stego-image formation using NPP-2 bit method}

\section{A. Selection of pixel positions of image}

Step I: Take the value of bit from array Arr[bit] to calculate total number of required pixels $(\mathrm{P})$. So, $\mathrm{P}=($ ceil (bit /3)).

Step II: Take the key $(\mathrm{K})$ and calculate the value of function: $\mathrm{F}(\mathrm{x}, \mathrm{y})=\mathrm{K}^{\mathrm{P}}$ [i.e. pow $(\mathrm{K}, \mathrm{P})$ ].

Step III: Store the exponential long double values one by one.

Step IV: Repeat Step II to Step III for $\mathrm{i}=(1$ to $\mathrm{p})$ and go to Step V.

Step V: Read the values as character up to "e" of the every line of the file and store it to file.

Step VI: Take most three significant digits to $\operatorname{Arr}_{\mathrm{x}}[\mathrm{p}]$, next three digits to array $\operatorname{Arr}_{\mathrm{y}}[\mathrm{p}]$ and last significant digit to $\operatorname{Arr}_{\mathrm{z}}[\mathrm{p}]$.

Step VII: Repeat Step V to Step VI up to end of the file.

Step VIII: Stop.

\section{B. Replacement of array elements with $R, G \& B$ values} of pixels

Step I: Calculate the width (w) and height (h) of the image.

Step II: Set $\mathrm{x}=\operatorname{Arr}_{\mathrm{x}}[\mathrm{P}]$ and $\mathrm{y}=\operatorname{Arr}_{\mathrm{y}}[\mathrm{P}]$. 
Step III: To select the pixel position into image, compare the value of $x$ and $y$ with the value of $w$ and $h$ (where addressable pixel position is $(0,0)$ to $(\mathrm{w}-1, \mathrm{~h}-1)$ ).

If $(\mathrm{x}>(\mathrm{w}-1))$ or $(\mathrm{y}>(\mathrm{h}-1))$ then

Set $P(x, y)=P(0+(x \%(w-1)),(0+(y \%(h-1)))$

Otherwise Set $\mathrm{P}(\mathrm{x}, \mathrm{y})=(\mathrm{x}, \mathrm{y})$.

Step IV: To select the bit position (b) of selected pixel i.e. with which bit the array data will be replaced. Set $\mathrm{z}$ $=\operatorname{Arr}_{\mathrm{z}}[\mathrm{p}]$.

i) If $(z \% 4=0)$ then $b=1^{\text {st }} \mathrm{LSB}$

ii) If $(z \% 4=1)$ then $b=2^{\text {nd }} \operatorname{LSB}$

iii) If $(z \% 4=2)$ then $b=3^{\text {rd }} L S B$, Otherwise $b=4^{\text {th }}$ LSB of each R, G \& B of a pixel.

Step V: A pair of data bits is replaced in corresponding and previous bit position and pixels are reformed.

Step VI: Repeat Step II to Step V for $\mathrm{i}=1$ to P.

Step VII: Stop.

\subsubsection{Creation of image and key shares using CVCV-8} Method

Step I: Create the image shares (IS $0^{-}$IS7) with presence $(\mathrm{P})$ or absence $(\mathrm{A})$ of Red $\left(\mathrm{IM}_{\mathrm{R}}\right)$, Green $\left(\mathrm{IM}_{\mathrm{G}}\right)$ and Blue $\left(\mathrm{IM}_{\mathrm{B}}\right)$ elements respectively.

Step II: Also the key shares $\left(\mathrm{KS}_{0}-\mathrm{KS}_{7}\right)$ are with presence $(\mathrm{P})$ or absence $(\mathrm{A})$ of key shares $\left(\mathrm{K}_{1}, \mathrm{~K}_{2}\right.$ and $\left.\mathrm{K}_{3}\right)$.

Step III: Stop.

\subsection{Decryption of the data bits from the image}

\subsubsection{Regain of replaced bits from the watermarked} image and formation of original content

Step I: Take any two possible shares of image IS $_{0^{-}}$ $\left.\mathrm{IS}_{7}\right)$ to reform the stego-image.

Step II: Also Take any two possible shares of key $\left(\mathrm{KS}_{0}-\mathrm{KS}_{7}\right)$ to reform the key $(\mathrm{K})$.

Step III: To get the pixel and bit position in R, G \& B of selected pixels go through Step I to Step VI of Algorithm 3.1.2.A and Step I to Step VI of Algorithm 3.1.2.B.

Step IV: Retrieving the encrypted bits from the selected bit positions of selected pixels store it to decrypted array from Darrlen[1] to Darrlen[bit] respectively.

Step V: To get the length repeat Step II to Step IV for $\mathrm{i}=1$ to 3 times (as every pixel contain three data bits) taking the key $\left(\mathrm{K}_{[0]}\right)$ and $\operatorname{ImgR} \mathrm{R}_{[0]}$.

Step VI: Taking data bits of Darr[1] as LSB and Darr[8] as MSB calculate the length (chlen) of message.

Step VII: Repeat Step VIII for $\mathrm{i}=1$ to 4 .

Step VIII: Retrieving the encrypted bits, store it to decrypted character array from Darr[1] to Darr[bit] respectively (where bit is the array length from characters).

Step IX: Taking data values from the decrypted array Darr[ ], LSB as Darr[8*i+1] and MSB as Darr[8*(i+1))] respectively. Repeat Step V to Step VIII to decrypt the data bits and convert to its equivalent ASCII- 8 character. Store the character to an array Msg[len].
Step X: Finally assemble the original message from the array $\mathrm{Msg}[\mathrm{len}]$.

Step XI: Stop.

\section{IMPLEMENTATION AND RESULT}

Let the message to be encrypt is "MULTIMEDIA". So the length of the message $=10=00001010$ ( 8 Bit Binary equivalent).

First the bits from length and then from text are being stored to the array Arr[bit] respectively as,

Table 2. Characters with Binary Equivalent

\begin{tabular}{|c|c|}
\hline Character & 8Bbit Binary Equivalent \\
\hline $\mathrm{M}$ & 01001101 \\
\hline $\mathrm{U}$ & 01010101 \\
\hline $\mathrm{L}$ & 01001100 \\
\hline $\mathrm{T}$ & 01010100 \\
\hline $\mathrm{I}$ & 01001001 \\
\hline $\mathrm{M}$ & 01001101 \\
\hline $\mathrm{E}$ & 01000101 \\
\hline $\mathrm{D}$ & 01000100 \\
\hline $\mathrm{I}$ & 01001001 \\
\hline $\mathrm{A}$ & 01000001 \\
\hline
\end{tabular}

Let the key $(\mathrm{K})=63 \mathrm{~A} 75 \mathrm{C}$.

The image size $=128 \times 128(\mathrm{w} \mathrm{x} \mathrm{h})$.

Number of effected pixel required for character $(\mathrm{p})=$ [ceil $(90 / 3)]=30$.

Calculate total $(\mathrm{t})=54+51+65+55+53+67=345$

Reminder $(\mathrm{n})=345 \% 8=1$.

In the table below the process of encryption of text data depending on key is described.

Table 3. Encryption Using MDE Method

\begin{tabular}{|c|c|}
\hline Bits after transposition & Bits after shifting \\
\hline 01110010 & 00111001 \\
\hline 10100011 & 11010001 \\
\hline$:$ & $:$ \\
\hline$:$ & $:$ \\
\hline 01110110 & 00111011 \\
\hline
\end{tabular}

From the Table 2 to Table 3 how the data bits are encrypted and in Table 4 how the encrypted data bits are embedded is described.

Table 4. Positions of Array Elements about the Image

\begin{tabular}{|c|c|c|c|c|}
\hline $\begin{array}{c}\text { Key, } \\
\text { i })\end{array}$ & Value & $\begin{array}{c}\text { Pixel } \\
\text { Position }\end{array}$ & $\begin{array}{c}\text { Bit } \\
\text { Position }\end{array}$ & Array Data \\
\hline 345,1 & $3450000 \mathrm{E}-$ & $(89,128)$ & LSB & $\begin{array}{c}\text { Earr[1-2] } \\
\text { Earr[3-4] } \\
\end{array}$ \\
04 & & & Earr[5-6] \\
\hline$:$ & $:$ & $:$ & $:$ & $:$ \\
\hline 345,1 & $2533486 \mathrm{E} 2$ & $(125,92)$ & $3^{\text {rd }}$ LSB & $\begin{array}{c}\text { Earr[85-86] } \\
\text { Earr[87-88] }\end{array}$ \\
& 22 & & & Earr[89-90] \\
\hline
\end{tabular}

In figure 3 the original and image shares are presented. In figure 4 the histogram for the cover image and stegoimage is given. 


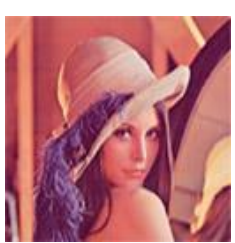

(a)

(d)

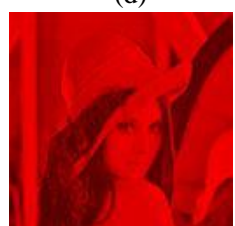

(g)

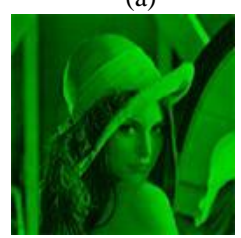

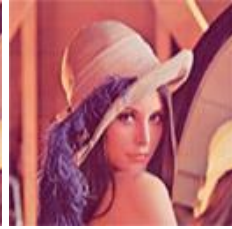

(b)

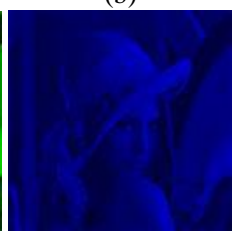

(e)

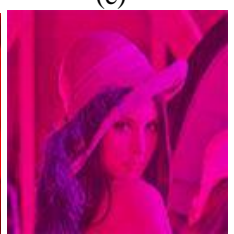

(h)

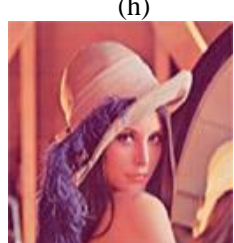

(j)

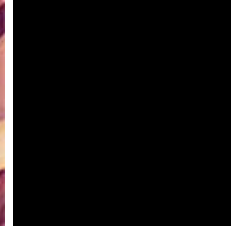

(c)

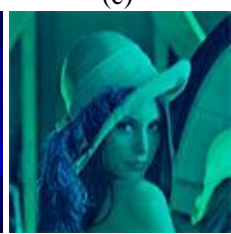

(f)

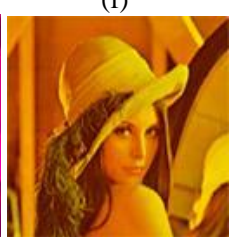

(i)

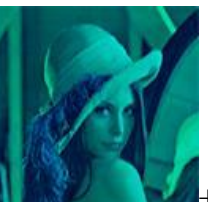

(a)

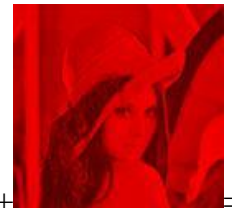

(b)

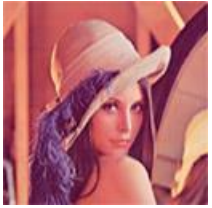

(c)
Fig.5. (a) (b) and (c) are the image subset IS4, IS5 and stego-image(SI)

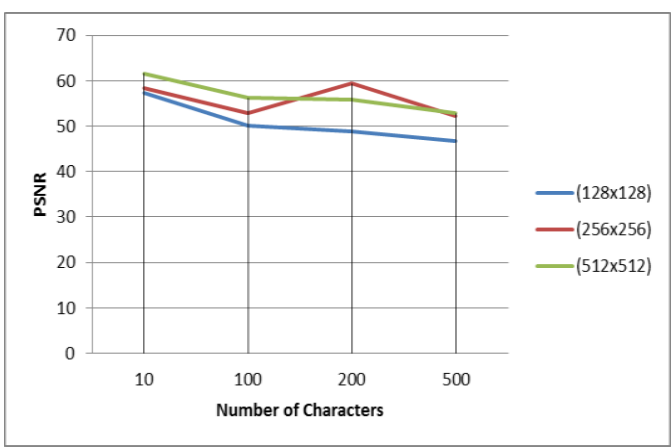

Fig. 6. Comparison of PSNR for image shares using NPP-2 bit method for LENA image

Table 5. PSNR of Image Shares using CVCI-8 Method

\begin{tabular}{|c|c|}
\hline Characteristics & CVCI-8 \\
\hline 1st image share PSNR & 26.6274 \\
\hline 2nd image share PSNR & 27.1404 \\
\hline 3rd image share PSNR & 27.2427 \\
\hline 4th image share PSNR & 27.8866 \\
\hline 5th image share PSNR & 28.5859 \\
\hline 6th image share PSNR & 30.0579 \\
\hline 7th image share PSNR & 30.2734 \\
\hline 8th image share PSNR & Infinity \\
\hline No. of Shares Required for & Any Two as Per \\
Decryption & Algorithm \\
\hline Reconstructed Image PSNR & Infinity \\
\hline Quality and Clarity & High \\
\hline Computational Complexity & Very High \\
\hline Hardware Implementation & High \\
\hline
\end{tabular}

Fig.3. (a) is the first original image, (b) the watermarked image, (c) (d) (e) (f) (g) (f) (i) and (j) image shares corresponding to subsets (Table 1.1) from ISO to IS8 respectively.

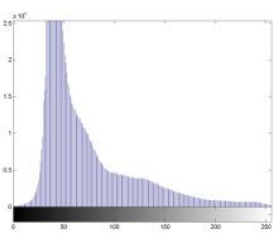

(a)

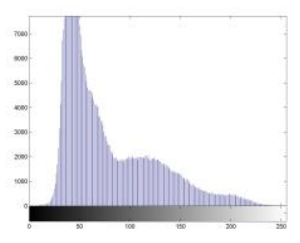

(b)
Fig.4. (a) and (b) the histogram of cover image, stego-image respectively

At the time of decryption let for $\mathrm{SI}_{24}\left(\left\{\mathrm{IS}_{3}, \mathrm{IS}_{4}\right\}\right)$, the union of subsets $\mathrm{IS}_{3}$ and $\mathrm{IS}_{4}$ make possible to produce the stego-image.

Table 6. Performance at a Glance for ISTI Method

\begin{tabular}{|c|c|c|c|c|c|}
\hline \multirow{2}{*}{ Images } & \multirow{2}{*}{$\begin{array}{c}\text { Image Similarity } \\
\text { Matrices }\end{array}$} & 10 & 100 & 200 & 500 \\
\cline { 2 - 6 } & PSNR & 57.2190 & 50.1686 & 48.8994 & 46.7911 \\
\hline \multirow{3}{*}{$\begin{array}{c}\text { LENA } \\
(128 x 128)\end{array}$} & MSE & 0.0152 & 0.3912 & 0.7019 & 1.8532 \\
\cline { 2 - 6 } & RMSE & 0.1233 & 0.6254 & 0.8337 & 1.3613 \\
\cline { 2 - 6 } & SSIM & 0.9999 & 0.9999 & 0.9998 & 0.9995 \\
\cline { 2 - 6 } & Cross Correlation & 1.0000 & 0.9999 & 0.9999 & 0.9999 \\
\cline { 2 - 6 } & UIQI & 0.9999 & 0.9999 & 0.9998 & 0.9995 \\
\cline { 2 - 6 } & Entropy & 7.2779 & 7.2786 & 7.2793 & 7.2803 \\
\cline { 2 - 6 } & K-L Divergence & $9.1256 \mathrm{E}-6$ & $9.8666 \mathrm{E}-5$ & $2.0388 \mathrm{E}-4$ & $5.2539 \mathrm{E}-4$ \\
\hline
\end{tabular}




\begin{tabular}{|c|c|c|c|c|c|}
\hline \multirow{8}{*}{$\begin{array}{l}\text { MONALISA } \\
(128 \times 128)\end{array}$} & PSNR & 54.1677 & 50.2087 & 48.7557 & 46.6074 \\
\hline & MSE & 0.0620 & 0.3840 & 0.7489 & 0.8029 \\
\hline & RMSE & 0.2490 & 0.6197 & 0.8659 & 0.8960 \\
\hline & SSIM & 0.9999 & 0.9998 & 0.9997 & 0.9997 \\
\hline & Cross Correlation & 0.9999 & 1.0000 & 1.0000 & 0.9999 \\
\hline & UIQI & 0.9999 & 0.9998 & 0.9997 & 0.9997 \\
\hline & Entropy & 6.5800 & 6.5807 & 6.5801 & 6.5809 \\
\hline & K-L Divergence & $1.6495 \mathrm{E}-5$ & $1.1866 \mathrm{E}-4$ & $2.5224 \mathrm{E}-4$ & $2.9737 \mathrm{E}-4$ \\
\hline \multirow{8}{*}{$\begin{array}{c}\text { LENA } \\
(256 \times 256)\end{array}$} & PSNR & 58.3020 & 52.7810 & 59.3981 & 52.3215 \\
\hline & MSE & 0.0092 & 0.1174 & 0.2220 & 0.0973 \\
\hline & RMSE & 0.0961 & 0.3427 & 0.4712 & 0.3125 \\
\hline & SSIM & 0.9999 & 0.9999 & 0.9999 & 0.9997 \\
\hline & Cross Correlation & 0.9999 & 0.9999 & 1.0000 & 0.9999 \\
\hline & UIQI & 0.9999 & 0.9999 & 0.9999 & 0.9997 \\
\hline & Entropy & 7.2735 & 7.2737 & 7.2740 & 6.5608 \\
\hline & K-L Divergence & $6.0120 \mathrm{E}-7$ & $1.2022 \mathrm{E}-5$ & $1.5636 \mathrm{E}-5$ & $1.3971 \mathrm{E}-5$ \\
\hline \multirow{8}{*}{$\begin{array}{l}\text { MONALISA } \\
(256 \times 256)\end{array}$} & PSNR & 57.9877 & 53.4006 & 53.1847 & 51.6239 \\
\hline & MSE & 0.0106 & 0.0883 & 0.0975 & 0.2001 \\
\hline & RMSE & 0.1033 & 0.2971 & 0.3123 & 0.4473 \\
\hline & SSIM & 0.9999 & 0.9999 & 0.9999 & 0.9999 \\
\hline & Cross Correlation & 0.9999 & 0.9999 & 0.9999 & 0.9999 \\
\hline & UIQI & 0.9999 & 0.9999 & 0.9999 & 0.9999 \\
\hline & Entropy & 6.5407 & 6.5408 & 6.5408 & 6.5410 \\
\hline & K-L Divergence & $1.0014 \mathrm{E}-6$ & $8.9826 \mathrm{E}-6$ & $1.8981 \mathrm{E}-5$ & $2.6238 \mathrm{E}-5$ \\
\hline \multirow{8}{*}{$\begin{array}{l}\text { LENA } \\
(512 \times 512)\end{array}$} & PSNR & 61.6574 & 56.1612 & 55.9398 & 52.8098 \\
\hline & MSE & $6.5697 \mathrm{E}-4$ & 0.0247 & 0.2742 & 0.1159 \\
\hline & RMSE & 0.0443 & 0.1573 & 0.1656 & 0.3404 \\
\hline & SSIM & 0.9999 & 0.9999 & 0.9999 & 0.9999 \\
\hline & Cross Correlation & 0.9999 & 0.9999 & 1.0000 & 0.9999 \\
\hline & UIQI & 0.9999 & 0.9999 & 0.9999 & 0.9999 \\
\hline & Entropy & 7.2881 & 7.2882 & 7.2881 & 7.2883 \\
\hline & K-L Divergence & $4.1259 \mathrm{E}-8$ & $4.6050 \mathrm{E}-7$ & $3.6156 \mathrm{E}-7$ & $2.1295 \mathrm{E}-6$ \\
\hline \multirow{8}{*}{$\begin{array}{l}\text { MONALISA } \\
(512 \times 512)\end{array}$} & PSNR & 61.1467 & 56.1969 & 54.7573 & 54.5438 \\
\hline & MSE & $8.3118 \mathrm{E}-4$ & 0.0243 & 0.0157 & 0.0521 \\
\hline & RMSE & 0.0499 & 0.1560 & 0.2174 & 0.2283 \\
\hline & SSIM & 0.9999 & 0.9999 & 0.9999 & 0.9999 \\
\hline & Cross Correlation & 1.0000 & 0.9999 & 0.9999 & 0.9999 \\
\hline & UIQI & 0.9999 & 0.9999 & 0.9999 & 0.9999 \\
\hline & Entropy & 6.7464 & 6.7463 & 6.7464 & 6.7464 \\
\hline & K-L Divergence & $6.2867 \mathrm{E}-7$ & $4.6102 \mathrm{E}-7$ & $1.0226 \mathrm{E}-6$ & $1.3922 \mathrm{E}-6$ \\
\hline
\end{tabular}

Table 7. Comparative Analysis of Our Proposed Method with Other Method

\begin{tabular}{|c|c|c|}
\hline Characteristics & Wang et al.[7] & CVCI-8 \\
\hline Types of Secret Sharing & $(2,2)$ & $(1,8)$ \\
\hline Work on & Halftone (black \& white) & Color Image \\
\hline Size of Input Image & $512 \times 512$ & $\begin{array}{c}\text { Any possible image } \\
\text { size }\end{array}$ \\
\hline Pixel Expansion & No & No \\
\hline Intensity Division & No & No \\
\hline Contrast Stretchment & No & No \\
\hline Extra Information Needed & Yes & No \\
\hline Codebook Needed & Yes & No \\
\hline Shares Generated on the Basis of & Blocks & Pixel (RGB value) \\
\hline
\end{tabular}




\section{ANALYSIS}

Here we have created a stego-image and then the image subsets using color components. We have used encryption technique but not any compression before the creation of array. Anybody may employ the compression at the time of creation of array. In that case, the length of array will be less and the strength of encryption will be higher than present. In addition the number of affected pixel will also be fewer. Here we have generated pixel positions depending on key value. Also key subsets have generated using key digits or characters. Here we have used six alphanumeric characters. Anybody may use only numerical digits and also with more digits or characters with variable length. As well may generate more subset of key. A pair of data bits are placed any five LSB position. Anybody may place less or more number of bits in any pixel position to each of $\mathrm{R}, \mathrm{G}$ and $\mathrm{B}$ color components. The number of targeted pixels proportionally varies to size of text. If the image size becomes large and size of text becomes less then it will be quite harder to differentiate the encrypted image from the original image.

\section{CONCLUSION}

Here we have used private key cryptographic technique to place the data bits (from both text and size of text) in arbitrary pixel positions about the entire image. Also we have generated random pixel positions depending on key. After that we generated image and key subsets from stego- image and key. Moreover, it produces the similar image using this method to see in naked eye. At the time of decryption only a proper combination of image and key subsets may produce the original stego -mage and key from which data can be extracted. After all, it will be quite impossible to find out the information from the stego-image.

\section{REFERENCES}

[1] Feng Liu, Chuankun Wu," Embedded Extended Visual Cryptography Schemes" , IEEE Transactions On Information Forensics And Security, Vol. 6, No. 2, June 2011, pp.307-322.

[2] Souvik Bhattacharyya, Goutam Sanyal "A Data Hiding Model with High Security Features Combining Finite State Machines and PMM method", International Journal of Electrical and Computer Engineering 5:2 2010, pp. 7885.

[3] Sabyasachi Samanta, Saurabh Dutta, Goutam Sanyal, "An Enhancement of Security on Image Applying Asymmetric Key Algorithm", International Journal of Computer Applications (0975 - 8887), Volume 25- No.5, July 2011, pp. 19-23.

[4] Shan-Chun Liu, Wen-Hsiang Tsai, "Line-Based CubismLike Image-A New Type of Art Image and its Application to Lossless Data Hiding "IEEE Transactions On Information Forensics And Security, Vol. 7, No. 5, October 2012, pp. 1148-1458.

[5] Chung-Ming Wang, Nan-I Wu, Chwei-Shyong Tsai , Min-Shiang Hwang, "A high quality steganographic method with pixel-value differencing and modulus function", The Journal of Systems and Software (2007), pp. 1-9.

[6] J. K. Mandal, Debashis Das, "Steganography Using Adaptive Pixel Value Differencing (APVD) of Gray Images through Exclusion of Overflow/Underflow", CS \& IT-CSCP 2012, pp. 93-102.

[7] Pradeep Kumar Sharma, Hari Mohan Singh" Visual Cryptography Scheme for Gray Scale Images based on Intensity Division" International Journal of Current Engineering and Technology, Vol.4, No.1 (Feb 2014) PISSN 2347 - 5161 pp.211-215.

[8] Dr. Ekta Walia, Payal Jain, An Analysis of LSB \& DCT based Steganography", Global Journal of Computer Science and Technology, Vol. 10, Issue 1 (Ver 1.0), April 2010, pp. 4-8.

[9] M. Abolghasemi, H. Aghaeinia, K. Faez, "Data Hiding Detection Based on DWT and Zernike Moments" 4th International Conference: Sciences of Electronic, Technologies of Information and Telecommunications, March 25-29, 2007 - TUNISIA.

[10] Linfeng Guo, Yan Meng,"PSNR-Based Optimization of JPEG Baseline Compression on Color Images", ICIP 2006, pp. 1145-1148.

[11] Atallah M. Al-Shatnawi, "A New Method in Image Steganography with Improved Image Quality", Applied Mathematical Sciences, Vol. 6, 2012, pp. 3907 - 3915.

[12] Himanshu Gupta, Vinod Kumar Sharma, "Role of Multiple Encryption in Secure Electronic Transaction", International Journal of Network Security \& Its Applications (IJNSA), Vol.3, No.6, November 2011, pp. 89-96.

[13] Pranam Paul, Saurabh Dutta, A K Bhattacharjee, "An Approach to ensure Security through Bit-level Encryption with Possible Lossless Compression", IJCSNS International Journal of Computer Science and Network Security, VOL.8 No.2, February 2008, pp. 291-299.

[14] InKoo Kang, Gonzalo R. Arce, Heung-Kyu Lee, "Color Extended Visual Cryptography Using Error Diffusion", IEEE Transactions on Image Processing, Vol. 20, No. 1, January 2011, pp. 132-145.

[15] Shunquan Tan, Bin Li, "Targeted Steganalysis of Edge Adaptive Image Steganography Based on LSB Matching Revisited Using B-Spline Fitting ", IEEE Signal Processing Letters, Vol. 19, No. 6, June 2012, pp. 336339.

\section{Authors' Profiles}

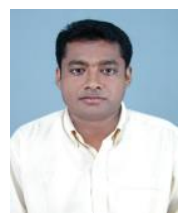

Sabyasachi Samanta is working as Assistant Professor at Dept. of IT, Haldia Institute of Technology Haldia, WB, and India. He has received M. Tech Degree in IT and currently pursuing $\mathrm{Ph}$. $\mathrm{D}$ at National Institute of Technology, Durgapur, WB, India. His main research interest includes watermarking, steganography and cryptography.

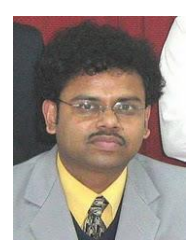

Saurabh Dutta is a professor in Dr. B. C. Roy Engineering College. He holds a Ph. D Degree in Coputer Science. His research domain is information security and cryptology. 


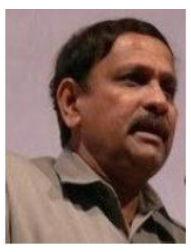

Gautam Sanyal is a member of the IEEE. He has received his B.E and M. Tech degree from National Institute of Technology (NIT), Durgapur, India. He has received Ph.D. (Engg.) from Jadavpur University, Kolkata, India, in the area of Robot Vision.

He possesses an experience of more than 25 years in the field of teaching and research. He has published nearly 68 papers in International and National Journals / Conferences. Three Ph. Ds (Engg.) have already been awarded under his guidance. At present he is guiding six $\mathrm{Ph}$. Ds scholars in the field of steganography, Cellular Network, High
Performance Computing and Computer Vision. He has guided over 10 PG and $100 \mathrm{UG}$ thesis. His research interests include Natural Language Processing, Stochastic modeling of network traffic, High Performance Computing, Computer Vision. He is presently working as a Professor in the department of Computer Science and Engineering and also holding the post of Dean (Students' Welfare) at National Institute of Technology, Durgapur, India.

How to cite this paper: Sabyasachi Samanta, Saurabh Dutta, Gautam Sanyal,"An Image Steganography-based Novel Approach to develop 8-Share Integrated Security Toolkit (ISTI-8)", IJIEEB, vol.7, no.3, pp.52-59, 2015. DOI: 10.5815/ijieeb.2015.03.08 